\title{
In Vivo Imaging of the Programmed Death Ligand 1 by ${ }^{18}$ F PET
}

\author{
Dinko E. González Trotter ${ }^{1}$, Xiangjun Meng ${ }^{1}$, Paul McQuade ${ }^{1}$, Daniel Rubins ${ }^{1}$, Michael Klimas ${ }^{1}$, Zhizhen Zeng ${ }^{1}$, \\ Brett M. Connolly ${ }^{1}$, Patricia J. Miller ${ }^{1}$, Stacey S. O'Malley ${ }^{1}$, Shu-An Lin ${ }^{1}$, Krista L. Getty ${ }^{2}$, Laurence Fayadat-Dilman ${ }^{3}$, \\ Linda Liang ${ }^{3}$, Elisabet Wahlberg ${ }^{4}$, Olof Widmark ${ }^{4}$, Caroline Ekblad ${ }^{4}$, Fredrik Y. Frejd ${ }^{4,5}$, Eric D. Hostetler ${ }^{1}$, \\ and Jeffrey L. Evelhoch ${ }^{1}$
}

${ }^{1}$ Translational Biomarkers Department, Merck \& Co., Inc., West Point, Pennsylvania; ${ }^{2}$ Screening and Protein Sciences Department, Merck \& Co., Inc., West Point, Pennsylvania; ${ }^{3}$ Biologics Discovery, Merck \& Co., Inc., Palo Alto, California; ${ }^{4}$ Affibody AB, Solna, Sweden; and ${ }^{5}$ Department of Immunology, Genetics and Pathology, Uppsala University, Uppsala, Sweden

Programmed death ligand 1 (PD-L1) is an immune regulatory ligand that binds to the T-cell immune check point programmed death 1. Tumor expression of PD-L1 is correlated with immune suppression and poor prognosis. It is also correlated with therapeutic efficacy of programmed death 1 and PD-L1 inhibitors. In vivo imaging may enable real-time follow-up of changing PD-L1 expression and heterogeneity evaluation of PD-L1 expression across tumors in the same subject. We have radiolabeled the PD-L1-binding Affibody molecule NOTA-Z $\mathrm{PD}_{\mathrm{P}-\mathrm{L} 1 \_}$with ${ }^{18} \mathrm{~F}$ and evaluated its in vitro and in vivo binding affinity, targeting, and specificity. Methods: The affinity of the PD-L1-binding Affibody ligand $\mathrm{Z}_{\mathrm{PD}-\mathrm{L}_{1} 1}$ was evaluated by surface plasmon resonance. Labeling was accomplished by maleimide coupling of NOTA to a unique cysteine residue and chelation of ${ }^{18} \mathrm{~F}-\mathrm{AlF}$. In vivo studies were performed in PD-L1positive, PD-L1-negative, and mixed tumor-bearing severe combined immunodeficiency mice. Tracer was injected via the tail vein, and dynamic PET scans were acquired for $90 \mathrm{~min}$, followed by $\gamma$-counting biodistribution. Immunohistochemical staining with an antibody specific for anti-PD-L1 (22C3) was used to evaluate the tumor distribution of PD-L1. Immunohistochemistry results were then compared with ex vivo autoradiographic images obtained from adjacent tissue sections. Results: NOTA-Z $Z_{\mathrm{PD}-\mathrm{L} 1 \_1}$ was labeled, with a radiochemical yield of $15.1 \% \pm 5.6 \%$, radiochemical purity of $96.7 \% \pm 2.0 \%$, and specific activity of $14.6 \pm 6.5 \mathrm{GBq} / \mu \mathrm{mol}$. Surface plasmon resonance showed a NOTA-conjugated ligand binding affinity of $1 \mathrm{nM}$. PET imaging demonstrated rapid uptake of tracer in the PD-L1-positive tumor, whereas the PD-L1-negative control tumor showed little tracer retention. Tracer clearance from most organs and blood was quick, with biodistribution showing prominent kidney retention, low liver uptake, and a significant difference between PD-L1-positive (percentage injected dose per gram $[\% \mathrm{ID} / \mathrm{g}]=2.56 \pm 0.33)$ and -negative $(\% \mathrm{ID} / \mathrm{g}=0.32 \pm$ $0.05)$ tumors $(P=0.0006)$. Ex vivo autoradiography showed excellent spatial correlation with immunohistochemistry in mixed tumors. Conclusion: Our results show that Affibody ligands can be effective at targeting tumor PD-L1 in vivo, with good specificity and rapid clearance. Future studies will explore methods to reduce kidney activity retention and further increase tumor uptake.

Received Feb. 26, 2017; revision accepted May 26, 2017.

For correspondence or reprints contact: Dinko E. González Trotter, Merk \& Co., Inc., 770 Sumneytown Pike, West Point, PA 19486.

E-mail: dinko.gonzalez@regeneron.com

Published online Jun. 6, 2017.

COPYRIGHT (C 2017 by the Society of Nuclear Medicine and Molecular Imaging.
Key Words: programmed death ligand 1; positron emission tomography; immuno-oncology; ${ }^{18} \mathrm{~F}-A I F-N O T A-Z_{P D-L 1 \_1}$

J Nucl Med 2017; 58:1852-1857

DOI: 10.2967/jnumed.117.191718

$\mathbf{N}$ egative regulators of $\mathrm{T}$ cell activity fulfill an important role in the prevention of autoimmune diseases. The check-point inhibitor ligand of programmed death 1 (PD-1), programmed death ligand 1 (PD-L1) (CD274), can be expressed constitutively by tumor cells, leading to an anergic $\mathrm{T}$ cell phenotype and avoidance of immune attack. Clinical correlation between PD-L1 tumor expression and anti-PD-1 therapy response has been established for certain malignancies (1), leading to the approval of an immunohistochemistry-based companion diagnostic for the antiPD-1 inhibitor pembrolizumab in non-small cell lung cancer $(2,3)$. Modulation of PD-L1 expression in tumors has been observed after therapeutic intervention and may be indicative of incipient mechanisms of immune resistance $(4,5)$.

A real-time in situ read of the expression of PD-L1 within all tumors in a patient may aid in understanding PD-L1's role in tumor response. A PD-L1 PET tracer would allow the interrogation of all metastatic sites of a patient in a single scanning session. Baseline and repeated scans would enable the evaluation of the impact of tumor PD-L1 expression heterogeneity on therapy response and the impact on PD-L1 expression of therapeutics used in combination with anti-PD-1/PD-L1 agents. PET and scintigraphic imaging against a variety of molecular targets have been performed in animal models and in the clinic using intact antibodies (6-11). The use of antibodies as tracers requires labeling with PET isotopes with half-lives of a few days (e.g., ${ }^{89} \mathrm{Zr}$ and ${ }^{124}$ I) due to slow clearance, with patients imaged several days after tracer injection. Smaller ligands tend to show faster blood clearance and can be labeled with the more widely available and shorter-half-life PET radioisotope ${ }^{18} \mathrm{~F}$ (109 min), enabling sameday patient imaging. Image quality may be improved compared with the longer-lived PET radioisotopes due to the larger positron emission fraction of ${ }^{18} \mathrm{~F}$ and greater injected doses because of a more favorable dosimetry profile.

Affibody molecules are based on a 58-amino-acid scaffold protein (12) from which high-affinity PD-L1 binders were selected by phage 

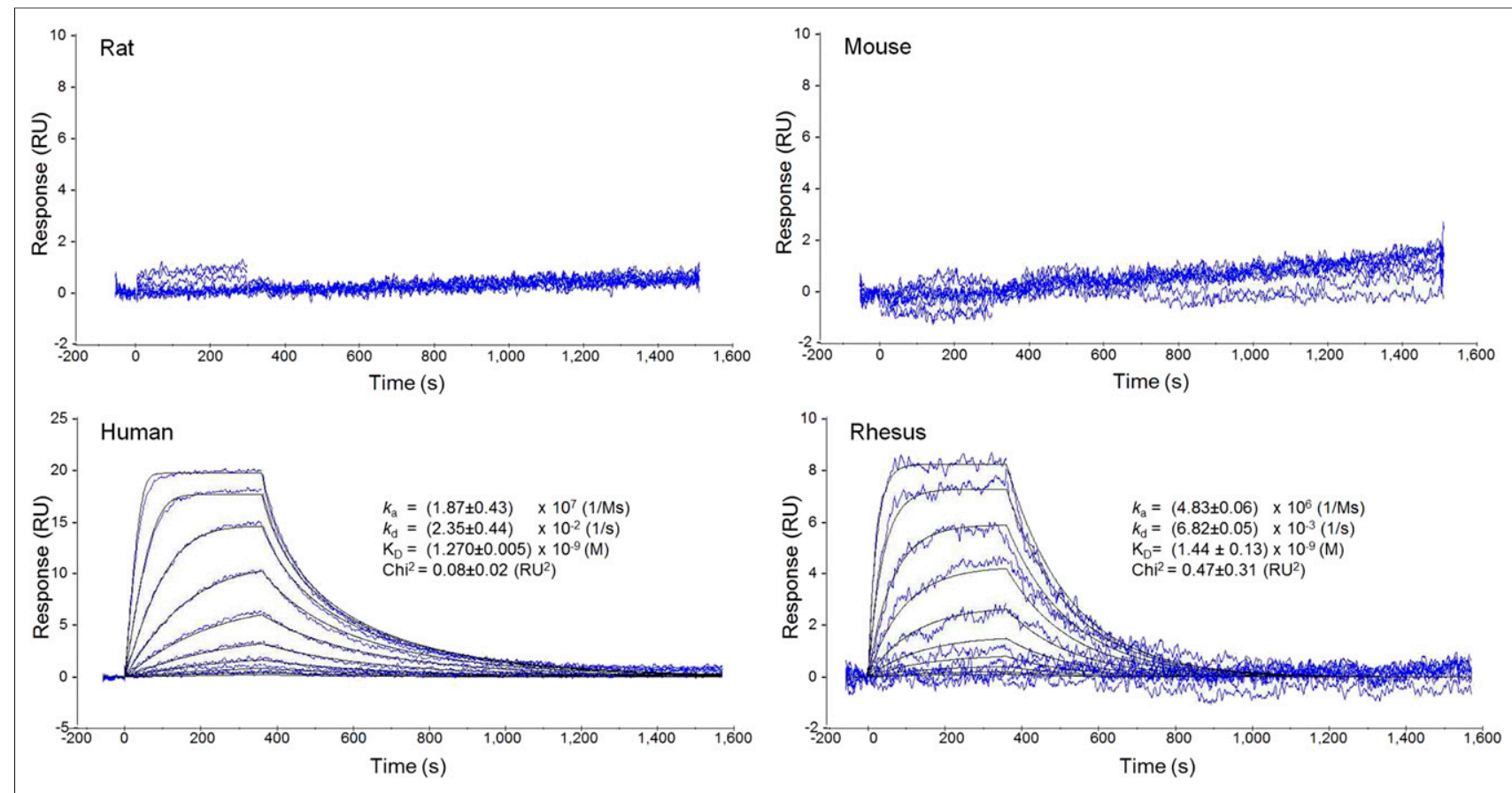

FIGURE 1. Surface plasmon resonance data taken by Biacore instrument for interaction of rat, mouse, human, and rhesus PD-L1 with NOTA-

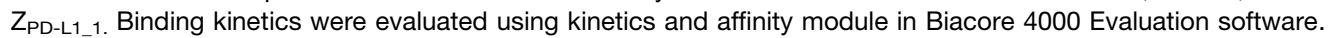

display. A unique engineered cysteine enables site-specific conjugation of linkers or chelators for PET and scintigraphic isotopes (13-16). Previous Affibody ligands have demonstrated rapid blood clearance and favorable in vivo imaging targeting properties in the clinic (17-19).

The present study evaluates the in vitro binding properties, in vivo targeting, ex vivo biodistribution, and ex vivo autoradiography compared with anti-PD-L1 immunohistochemistry of an ${ }^{18} \mathrm{~F}-$ labeled anti-PD-L1 Affibody molecule. Results demonstrate fast tracer blood clearance and high in vivo targeting specificity for PD-L1 expressed in xenograft tumors.

\section{MATERIALS AND METHODS}

\section{Production of ZPD L1_1}

E. coli T7E2 cells (GeneBridges GmBH) were transformed with plasmids containing the gene fragment encoding the PD-L1-targeting Affibody ligand with an $\mathrm{N}$ terminal $6 \times$ histidine tag, referred to as His-ZPD L1_1, or a C terminal cysteine residue, referred to as ZPD L1_1. Cells were cultivated at $37^{\circ} \mathrm{C}$ in TSB-YE medium and protein expression was induced with IPTG.

ZPD L1_1 was purified by anion exchange (Q Sepharose Fast Flow resin; GE Healthcare) followed by reversed-phase chromatography (SOURCE 15 RPC; GE Healthcare) and the disulfides were reduced with $20 \mathrm{mM}$ DTT for $1 \mathrm{~h}$ at room temperature prior to each purification step. Buffer exchange to $20 \mathrm{mM}$ HEPES, $1 \mathrm{mM}$ EDTA (pH 7.2) was carried out using HiPrep 26/10 columns (GE Healthcare). Finally, ZPD-L1_1 was purified on EndoTrap ${ }^{\circledR}$ red columns (Hyglos) to ensure low endotoxin content.

His-ZPD L1_1 was purified by Immobilized Metal ion Affinity Chromatography using a His-GraviTrap IMAC column (GE Healthcare). Buffer exchange to phosphate-buffered saline (PBS) $(10 \mathrm{mM}$ phosphate, $137 \mathrm{mM} \mathrm{NaCl}, 2.68 \mathrm{mM} \mathrm{KCl}, \mathrm{pH}$ 7.4) was performed using PD-10 desalting columns (GE Healthcare). The thermal stability of ZPD L1_1 was analyzed by circular dichroism spectroscopy.

\section{DOTA Conjugation of MEB037.22C3.138 (22C3)}

Two milligrams of the human PD-L1-specific murine mAb 22C3 (Merck \& Co., Inc.) and $0.11 \mathrm{mg}(0.13 \mu \mathrm{mol})$ of DOTA-NHS were stirred together for $18 \mathrm{~h}$ at $4^{\circ} \mathrm{C}$ in Na2HPO4 (0.1 M, pH 8.5). The reaction was then purified via a Centricon YM-100 Centrifugal Filter (Millipore Corp.), with the purified DOTA-22C3 collected in ammonium citrate and stored in $100-\mu \mathrm{L}$ aliquots at $-70^{\circ} \mathrm{C}$.

\section{${ }^{64} \mathrm{Cu}$-DOTA-22C3}

Two microliters of a ${ }^{64} \mathrm{CuCl} 2$ solution in $0.1 \mathrm{M} \mathrm{HC}$ were added to $25 \mu \mathrm{g}$ of DOTA-22C3 in $100 \mu \mathrm{L}$ of ammonium citrate (0.1M, pH 5.5). After the reaction mixture was incubated at $37^{\circ} \mathrm{C}$ for $30 \mathrm{~min}$ and allowed to cool for $5 \mathrm{~min}$, diethylenetriaminepentaacetic acid $(5 \mu \mathrm{L}$, $10 \mathrm{mM}$ ) was added. The ${ }^{64} \mathrm{Cu}$-DOTA-22C3 was purified via a BioSpin 30 column (Bio-Rad), with radiochemical purity determined using a Waters 2795 HPLC system equipped with a Waters 2998 UV detector and $\beta$-RAM Model 4 radio-HPLC detector using a TSKgel G3000SWXL size-exclusion column.

\section{Homogenate Binding Studies}

Tissue homogenates at $6-8 \mathrm{mg} / \mathrm{mL}$ of wet tissue weight were preincubated in Skatron tube strips (SK15776) with assay buffer, or unlabeled $22 \mathrm{C} 3$ antibody $(0.5 \mu \mathrm{M})$ at room temperature for $30 \mathrm{~min}$ in assay buffer (PBS, pH 7.5, 1:1,000 protease inhibitor, $0.1 \%$ bovine serum albumin). Nondisplaceable binding of ${ }^{64} \mathrm{Cu}$-DOTA- $22 \mathrm{C} 3$ was defined using self-block ( $0.5 \mu \mathrm{M}$ unlabeled affibody). ${ }^{64} \mathrm{Cu}$-DOTA$22 \mathrm{C} 3$ concentrations were tested from $0.1 \mathrm{nM}$ to $10 \mu \mathrm{M}$. The assay tubes were incubated at room temperature for $195 \mathrm{~min}$. Following incubation, the reaction mixtures were transferred by a Skatron Combi cell harvester onto Skatron GF/C filters (SK11731) soaked in $0.3 \%$ PEI for $30 \mathrm{~min}$ at $4^{\circ} \mathrm{C}$ before use. The filters were promptly washed with ice-cold wash buffer (PBS, pH 7.5) 3 times, punched into Pico Pro vials, and then counted in a gamma counter using the ${ }^{64} \mathrm{Cu} 30$-s protocol. All data were analyzed by nonlinear fit (1-site binding) model using Prism software (GraphPad Software). 
TABLE 1

Radiochemical Purity, Specific Activity, and Radiochemical Yield of ${ }^{18}$ F-AIF-NOTA-Z ${ }_{\text {PD-L1_1 }}$ and ${ }^{64} \mathrm{Cu}-\mathrm{DOTA}-22 \mathrm{C3}$

\begin{tabular}{|c|c|c|}
\hline $\begin{array}{c}\text { Radiotracer } \\
\text { characteristic }\end{array}$ & ${ }^{18} \mathrm{~F}-A I F-N O T A-Z_{\text {PD-L1_1 }}$ & ${ }^{64} \mathrm{Cu}$-DOTA-22C3 \\
\hline $\begin{array}{l}\text { Percentage } \\
\text { radiochemical } \\
\text { purity }\end{array}$ & $96.7 \pm 2.0$ & 100 \\
\hline $\begin{array}{c}\text { Specific activity } \\
(\mathrm{GBq} / \mu \mathrm{mol})\end{array}$ & $14.6 \pm 6.5$ & $44.5 \pm 19.9$ \\
\hline $\begin{array}{l}\text { Percentage } \\
\text { radiochemical } \\
\text { yield }\end{array}$ & $15.1 \pm 5.6$ & $49.6 \pm 12.3$ \\
\hline
\end{tabular}

Data are mean $\pm \mathrm{SD}(n=3)$.

\section{Immunohistochemistry}

Slides containing sections adjacent to those used for ex vivo autoradiography were fixed in acetone/ethanol (3:1). After incubations with a monoclonal anti-PD-L1 antibody (22C3) followed by antimouse IgG polymer (IH-8061; ImmunoBioScience Corp.) and DAB chromogen (Dako Corp.), the slides were counterstained with hematoxylin. 22C3 anti-PD-L1 immunohistochemical staining of SUDHL6 and LOX xenograft tumor samples showed absence of signal in the negative control tumor, whereas clear membranous and some cytoplasmic cell staining could be observed in the LOX sample (Supplemental Fig. 1; supplemental materials are available at http://jnm. snmjournals.org)

\section{Cell Lines}

The LOX malignant melanoma cell line was selected because it expresses PD-L1 constitutively (20). The SUDHL6 lymphoma cell line was chosen as a negative control because it is reported not to express PD-L1 (21). The mixed tumor model was intended to demonstrate the specificity of tracer distribution ex vivo, within the same subject in a tumor mass with a common blood supply. The expression level of PD-L1 by the LOX cell line was within the median of the expression levels evaluated from melanoma tumor samples (data not shown).

\section{NOTA Conjugation of $\mathbf{Z}_{\mathrm{PD}-\mathrm{L1} \text { _1 }}$}

To a 4-(2-hydroxyethyl)-1-piperazineethanesulfonic acid (HEPES)ethylenediaminetetraacetic acid (EDTA) solution containing $5 \mathrm{mg}$ of
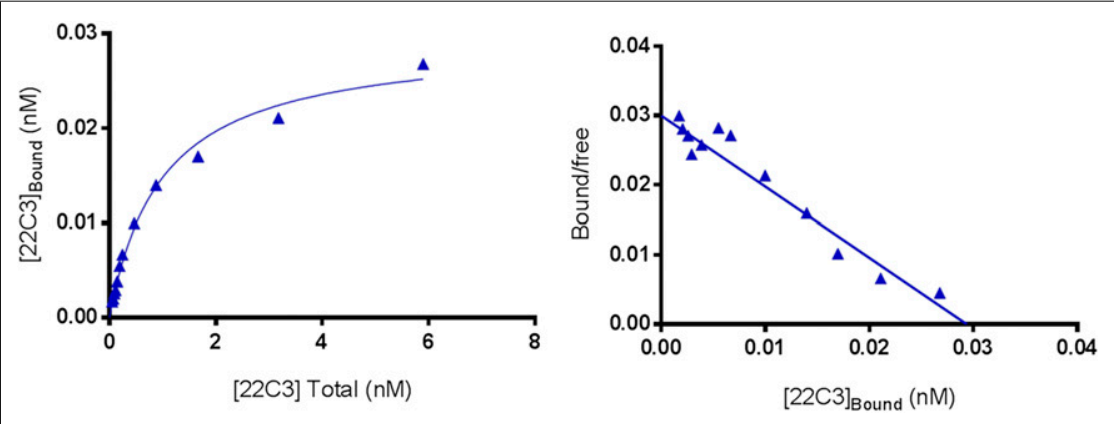

FIGURE 2. Equilibrium saturation curve (left) and Scatchard plot (right) for ${ }^{64} \mathrm{Cu}-\mathrm{DOTA}-22 \mathrm{C} 3$ binding in LOX tumor homogenates, showing saturable binding suggestive of binding to single target population.
$\mathrm{Z}_{\mathrm{PD}-\mathrm{L} 1 \text { 1 }}$ were added 3 equivalents of tris(2-carboxyethyl)phosphine in $0.5 \mathrm{~mL}$ of $0.2 \mathrm{M}$ ammonium acetate buffer ( $\mathrm{pH} 7.0$ ), which had been degassed 3 times. The reaction was kept at room temperature for $60 \mathrm{~min}$ before being transferred to an Ultracel $3 \mathrm{~K}$ Centrifugal Filter and centrifuged at 4,000 rpm for $90 \mathrm{~min}$. The solution was then rinsed with $1 \mathrm{~mL}$ of $0.2 \mathrm{M}$ ammonium acetate buffer and the reduced Affibody molecule transferred to a second reaction vessel in $2 \mathrm{~mL}$ of oxygen-free $0.2 \mathrm{M}$ ammonium acetate buffer ( $\mathrm{pH} 7.0)$. NOTA-maleimide $(4 \mathrm{mg})$ in $0.5 \mathrm{~mL}$ of $0.2 \mathrm{M}$ ammonium acetate buffer $(\mathrm{pH} 7.0)$ was added and the reaction vessel purged with argon and heated to $40^{\circ} \mathrm{C}$ for $3 \mathrm{~h}$, at which point the reaction mixture was transferred to a Ultracel $3 \mathrm{~K}$ Centrifugal Filter and centrifuged for $90 \mathrm{~min}$ at 4,000 rpm. After the flow-through was discarded and $2 \mathrm{~mL}$ of milliQ water were added, the mixture was again centrifuged for $90 \mathrm{~min}$ and the flow-through discarded. Purified

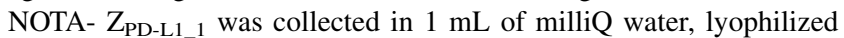
and stored at $-70^{\circ} \mathrm{C}$ before use. The purity of the final product was determined via LC/MS.

\section{${ }^{18}$ F-AIF-NOTA-Z}

A cartridge containing ${ }^{18} \mathrm{~F}$-fluoride was first washed with $1.5 \mathrm{~mL}$ of ultrapure water and then ${ }^{18} \mathrm{~F}$-fluoride was eluted with $1.0 \mathrm{~mL}$ of $0.4 \mathrm{M}$ $\mathrm{KHCO}_{3}$. One hundred microliters of the eluted ${ }^{18} \mathrm{~F}$-fluoride solution were added to a stem vial charged with $10 \mu \mathrm{L}$ of acetic acid, $50 \mu \mathrm{L}$ of $\mathrm{AlCl}_{3}(2 \mathrm{mM}$ in $0.1 \mathrm{M} \mathrm{NaOAc}$ buffer, $\mathrm{pH} 4)$, and $125 \mu \mathrm{L}$ of NaOAc $(0.1 \mathrm{M}, \mathrm{pH} 4)$. The solution was incubated for $2 \mathrm{~min}$ at room temperature before $1 \mathrm{mg}$ of NOTA-Z $\mathrm{PD}_{\mathrm{PD} \text { 1_1 }}$ in $400 \mu \mathrm{L}$ of a 1:1 solution of acetonitrile and $\mathrm{NaOAc}(0.1 \mathrm{M}, \mathrm{pH} 4)$ was added and then heated to $100^{\circ} \mathrm{C}$ for $15 \mathrm{~min}$. It was then transferred to a vial containing $0.7 \mathrm{~mL}$ of $0.1 \%$ formic acid, mixed and purified by high-performance liquid chromatography (Xselect CSH C18 [Waters]; $250 \times 10 \mathrm{~mm}, 130 \mu \mathrm{m}$ ) using a gradient of $10 \%-30 \% \mathrm{MeCN}$ in $0.1 \%$ formic acid over $15 \mathrm{~min}$ at a flow rate of $5 \mathrm{~mL} / \mathrm{min}$. The peak corresponding to ${ }^{18} \mathrm{~F}-\mathrm{AlF}$ -

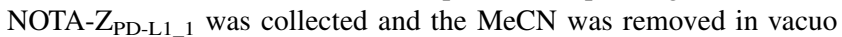
and transferred to a sterile vial using physiologic saline as a rinse to yield ${ }^{18}$ F-AlF-NOTA-Z ${ }_{\text {PD-L1_1 }}$. Specific activity and radiochemical purity were determined via a Waters Acquity LC/MS system and a $\beta$-RAM Model 4 radio-high-performance liquid chromatography (HPLC) detector (IN/US Systems).

\section{Animal Models}

All procedures involving animals were reviewed and approved by the Institutional Animal Care and Use Committee at Merck. Female severe combined immunodeficiency Beige mice (6-8 wk old) were ordered from Charles River Laboratories and housed in a temperatureand humidity-controlled room and on regular diet. LOX-IMVI (LOX) or SUDHL6 (American Type Culture Collection) cells were cultured in complete growth medium containing RPMI 1640 with $10 \%$ fetal bovine serum at $37^{\circ} \mathrm{C}$ with $5 \% \mathrm{CO}_{2}$. The growth medium was changed 2 or 3 times per week, and the cells were subcultured at a ratio of 1:10 when needed. Tumors were implanted at the right shoulder with a subcutaneous injection of $1 \times 10^{6}$ LOX cells in $100 \mu \mathrm{L}$ of phosphatebuffered saline or $10 \times 10^{6}$ SUDHL6 cells in $100 \mu \mathrm{L}$ of phosphate-buffered saline plus growth factor-reduced Matrigel (1:1). The mice were used for small-animal PET and ex vivo studies about 5-7 d and $3 \mathrm{wk}$ after the injection of LOX and SUDHL6 cells, respectively, when the tumors reached a mass of 100-400 mg.

For the development of the mixture of LOX and SUDHL6 tumors, the LOX cells were injected into the SUDHL6 tumors, 


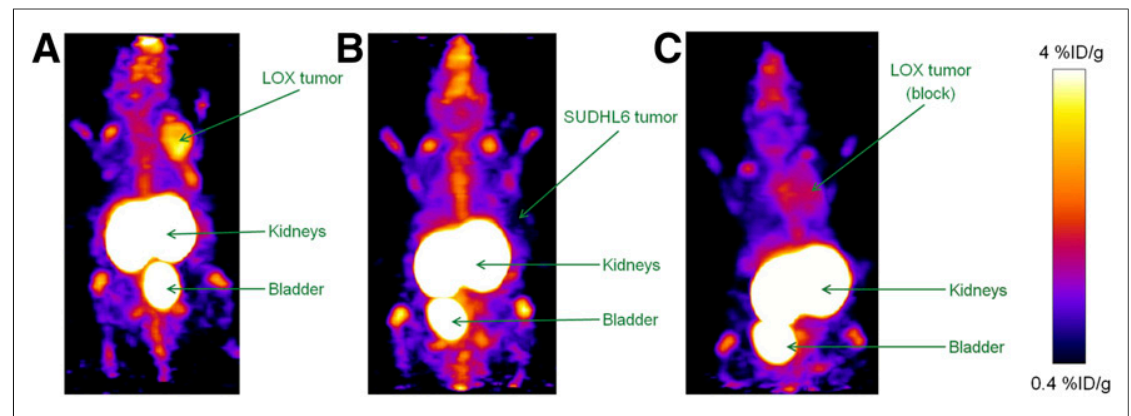

FIGURE 3. Representative PET maximum-intensity-projection images from 30 to 90 min after ${ }^{18} \mathrm{~F}-\mathrm{AIF}-\mathrm{NOTA}-\mathrm{Z}_{\mathrm{PD}-\mathrm{L} 1{ }_{1} 1}$ administration are shown for mouse xenograph model with LOX tumor $(\mathrm{A})$, SUDHL6 tumor (B), and LOX tumor after block with $400 \mu \mathrm{g}$ of NOTA-Z $\mathrm{PD}_{\mathrm{PD} \text { L1_1 }}(\mathrm{C})$. Tumor, kidneys, and bladder are indicated by arrows, and color scale represents $\% \mathrm{ID} / \mathrm{g}$ tissue. image plate scanner (Fujifilm Medical Systems), and the resulting images were processed with MCID software (Imaging Research, Inc.).

\section{RESULTS}

\section{Affibody Molecule Characterization}

Surface plasmon resonance measurements were used to assess the binding of NOTA-Z $Z_{\text {PD-L1_1 }}$ to mouse, rat, rhesus, and human PD-L1. Although NOTA-Z showed no measurable binding to either mouse or rat PD-L1, it was determined to have a ligand binding affinity $\left(\mathrm{K}_{\mathrm{D}}\right)$ of $1 \mathrm{nM}$ against both human and rhesus PD-L1 (Fig. 1). which were inoculated about 2 wk early. About 1 wk after implantation of LOX cells, mice were imaged and the tumors were harvested for histologic and autoradiographic studies.

\section{PET Data Acquisition and Ex Vivo Biodistribution Measurements}

Mice were anesthetized with isoflurane (4\%-5\% induction, $1 \%-3 \%$ maintenance), prepared with tail vein catheters, and placed in a dedicated small-animal PET scanner (microPET Focus220; Siemens Preclinical Solutions). A $20-\mathrm{min}$ transmission scan with a ${ }^{57} \mathrm{Co}$ source was obtained to correct for photon attenuation and scatter. Then 0.2-0.6 $\mathrm{MBq}$ of ${ }^{18} \mathrm{~F}$-AlF-NOTA-Z $\mathrm{PD}-\mathrm{L} 1 \_1_{\text {were }}$ administered via the tail vein, and PET list-mode data were collected for $90 \mathrm{~min}$. Immediately after PET acquisition, the mice were euthanized, and the tumor, heart, lung, spleen, liver, kidneys, blood, plasma, and muscle were collected and measured using a $\gamma$-counter (PerkinElmer). For each mouse, biodistribution measurements were converted into units of percentage injected dose per gram $(\% \mathrm{ID} / \mathrm{g})$.

\section{Ex Vivo Autoradiography}

The SUDHL6/LOX xenograft tumors removed after imaging were bisected and immediately frozen. Replicate sets of $14-\mu m$-thick cryosections were prepared and adhered to glass slides. For autoradiography, slides were air dried at room temperature, apposed to a phosphor imaging plate (TR 2025; Fujifilm Medical Systems), and exposed overnight. The plates were scanned on a BAS-5000 phosphor

\section{Radiolabeling and Homogenate Binding Studies}

${ }^{18}$ F-AlF-NOTA-Z ${ }_{\text {PD-L1_1 }}$ and ${ }^{64} \mathrm{Cu}$-DOTA-22C3 were obtained with high radiochemical purity, specific activity, and radiochemical yield (Table 1). The concentration of PD-L1 in LOX tumors was evaluated quantitatively through ${ }^{64} \mathrm{Cu}$-DOTA22C3 homogenate binding studies to understand the potential for ${ }^{18} \mathrm{~F}-\mathrm{AlF}-\mathrm{NOTA}-\mathrm{Z}_{\mathrm{PD}-\mathrm{L} 1 \text { 1_1 }}$ to saturate the target in vivo. ${ }^{64} \mathrm{Cu}$-DOTA-22C3 antibody exhibited displaceable and saturable binding in LOX tumor xenografts expressing PD-L1. As shown by Scatchard analysis, ${ }^{64} \mathrm{Cu}$-DOTA-22C 3 bound to 1 site with high affinity $\left(\mathrm{K}_{\mathrm{D}}=1.0 \mathrm{nM}\right)$ and good concentration of available target $\left(K_{D}=5\right)$ (Fig. 2). The concentration of PD-L1 in LOX tumor was approximately $4.9 \mathrm{nM}$.

\section{PET Data Acquisition and Ex Vivo Biodistribution Measurements}

Representative PET images after ${ }^{18} \mathrm{~F}-\mathrm{AlF}-\mathrm{NOTA}-\mathrm{Z}_{\mathrm{PD}-\mathrm{L} 1 \_1}$ injection are shown in Figure 3, demonstrating highest uptake in the kidneys and bladder. LOX tumors could be clearly seen whereas SUDHL6 tumors were not visible. Ex vivo biodistribution measurements (Fig. 4; Table 2) at 90 min after injection agreed with PET images, and ${ }^{18} \mathrm{~F}-\mathrm{AlF}-\mathrm{NOTA}-\mathrm{Z}_{\mathrm{PD}-\mathrm{L} 1 \_1}$ uptake was much higher in LOX tumors than SUDHL6 tumors (Fig. 4). Tissue-to-blood ratios for nontumor tissues in LOX and SUDHL6 mice did not differ. Blocking with $400 \mu \mathrm{g}$ of

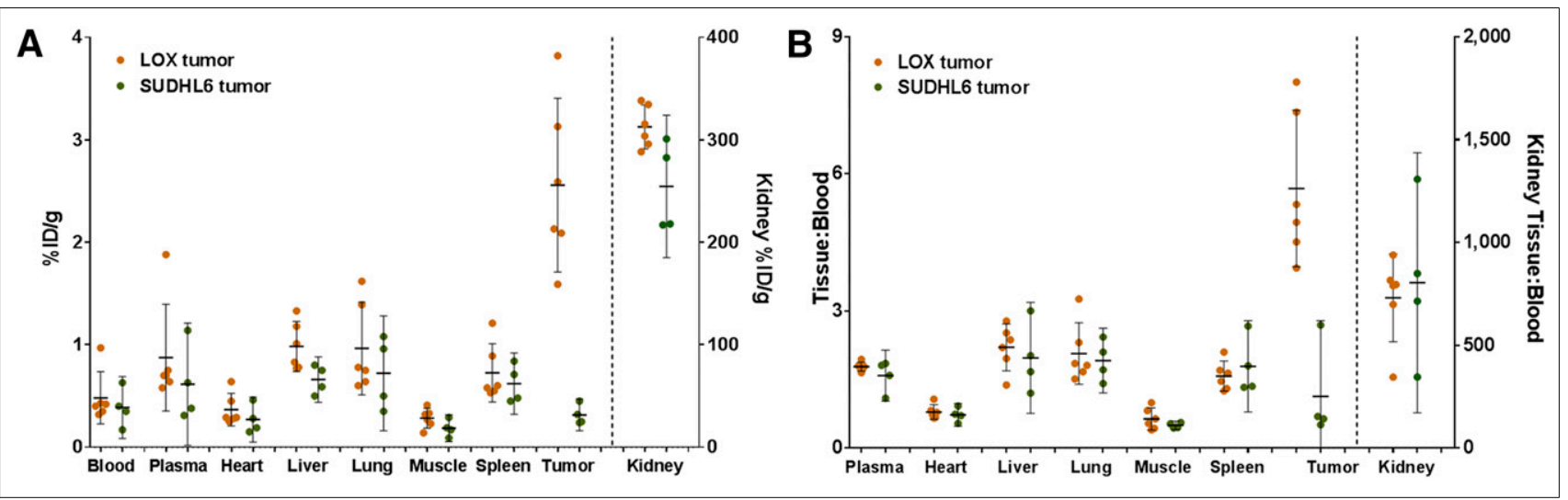

FIGURE 4. Ex vivo biodistribution results for LOX and SUDHL6 mouse xenograph models are displayed in units of \%ID/g (A) and tissue-to-blood ratio (B). Horizontal lines indicate arithmetic mean, and error bars represent $95 \%$ confidence interval. Vertical axis is on left for all tissues on left of vertical dashed line; vertical axis for kidney is on right because of significantly higher tracer level. 
TABLE 2

Ex Vivo Biodistribution Results for LOX and SUDHL6 Mouse Xenograph Models 90 Minutes After Injection

\begin{tabular}{lcc}
\hline $\begin{array}{c}\text { Organ or } \\
\text { tissue }\end{array}$ & $\begin{array}{r}\text { LOX tumor-bearing } \\
\text { SCID mice }(n=6)\end{array}$ & $\begin{array}{r}\text { SUDHL6 tumor-bearing } \\
\text { SCID mice }(n=4)\end{array}$ \\
\hline Blood & $0.48 \pm 0.10$ & $0.39 \pm 0.09$ \\
\hline Plasma & $0.87 \pm 0.20$ & $0.62 \pm 0.19$ \\
Heart & $0.37 \pm 0.06$ & $0.27 \pm 0.07$ \\
Kidney & $312.69 \pm 8.34$ & $254.59 \pm 21.80$ \\
Liver & $0.98 \pm 0.10$ & $0.66 \pm 0.07$ \\
Lung & $0.96 \pm 0.18$ & $0.72 \pm 0.18$ \\
Muscle & $0.28 \pm 0.04$ & $0.18 \pm 0.04$ \\
Spleen & $0.73 \pm 0.11$ & $0.62 \pm 0.09$ \\
Tumor & $2.56 \pm 0.33$ & $0.32 \pm 0.05$ \\
\multicolumn{3}{l}{} \\
\hline SCID = severe combined immunodeficiency. \\
Data are mean \pm SEM (\%ID/g).
\end{tabular}

NOTA-Z $Z_{\text {PD-L1_1 }}$ caused a reduction in ${ }^{18}$ F-AlF-NOTA-Z $Z_{\text {PD-L1_1 }}$ uptake in LOX tumors and also changed the biodistribution, including faster clearance as indicated by reduced blood uptake at $90 \mathrm{~min}$.

Prominent renal clearance and retention were observed in all mice. Blood clearance was rapid, with tumor-to-blood $\% \mathrm{ID} / \mathrm{g}$ ratios in excess of 5:1 achieved $90 \mathrm{~min}$ after injection. The \%ID/g ratio of LOX tumors compared with SUDHL6 was also high at 7:1. Uptake in most nontumor tissues was low, with the liver showing the highest uptake $(\% \mathrm{ID} / \mathrm{g}=0.98 \pm$ $0.10)$ after the kidneys $(\% \mathrm{ID} / \mathrm{g}=313 \pm 8)$ in LOX tumorbearing mice.

\section{Ex Vivo Autoradiography and Immunohistochemistry}

Comparison of the distribution of ex vivo tracer signal and immunohistochemistry in a mixed LOX and SUDHL6 tumor provides an additional test of specificity at a more detailed scale than traditional tissue biodstribution. Ex vivo autoradiography studies of mixed SUDHL6/LOX xenograft tumors showed heterogeneous uptake of ${ }^{18} \mathrm{~F}-\mathrm{AlF}-\mathrm{NOTA}-\mathrm{Z}_{\mathrm{PD}-\mathrm{L} 1 \_1}$. These areas of
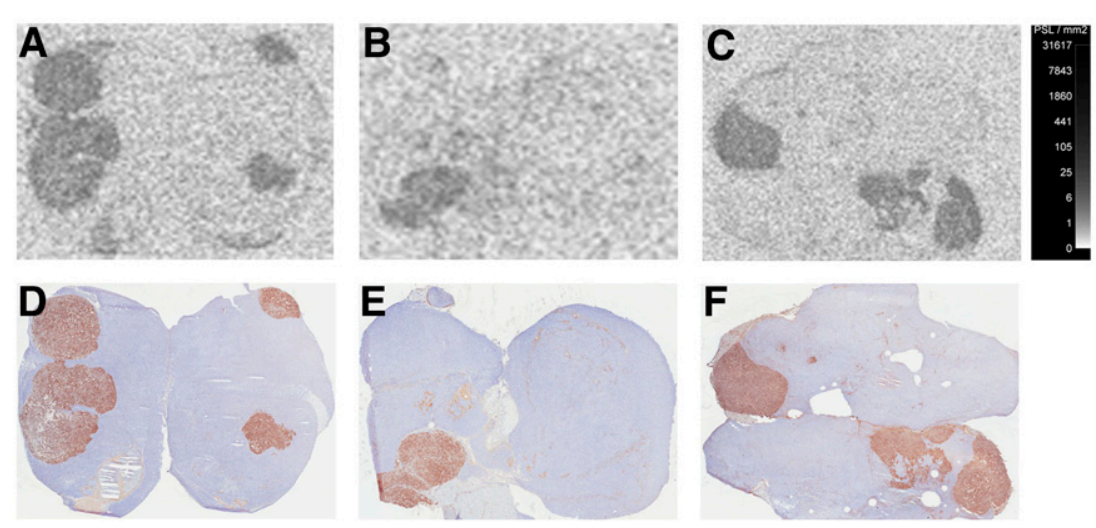

FIGURE 5. Ex vivo autoradiography $(\mathrm{A}-\mathrm{C})$ and $22 \mathrm{C} 3$ immunohistochemistry (D-F) of mixed SUDHL6/LOX xenograft tumors after ${ }^{18} \mathrm{~F}-\mathrm{AIF}-\mathrm{NOTA}-\mathrm{Z}_{\mathrm{PD}-\mathrm{L} 1 \_1}$ administration. Note close spatial agreement between areas of ${ }^{18} \mathrm{~F}-\mathrm{AlF}-\mathrm{NOTA}-\mathrm{Z}_{\mathrm{PD}-\mathrm{L} 1 \_1}$ activity and $22 \mathrm{C} 3$ staining of PD-L1. high uptake corresponded to the LOX tumor cell areas showing signal in 22C3 immunohistochemistry (Fig. 5).

\section{DISCUSSION}

PD-L1 is expressed in several malignancies and is associated with poor prognosis in gastric, esophageal, ovarian, pancreatic, and urothelial cancers $(22,23)$. Immunotherapeutic use of PD-1 and PD-L1 inhibitors has resulted in durable remissions in renal carcinoma, lung cancer, melanoma, and bladder cancer $(1,3,24,25)$. Tumor PD-L1 expression assessed by immunohistochemistry is correlated with response to anti-PD-1 and PD-L1 therapies $(1,2,4)$.

Tumor PD-L1 expression can change over time as a result of therapeutic interventions, such as BRAF inhibitors, chemotherapeutic agents, IFN- $\gamma$, and TNF- $\alpha$ (5). Repeated biopsy poses difficulties in obtaining tumor PD-L1 readings over time, or for several metastatic sites simultaneously. An ${ }^{18}$ F-labeled fast-clearing PD-L1targeting PET tracer would enable whole-body, real-time evaluation of PD-L1 tumor expression. Longitudinal noninvasive evaluation of tumor changes in PD-L1 expression may provide a useful tool for pharmacodynamic evaluation of immunotherapeutic intervention and identification of therapies that increase tumor immunogenicity and may increase effectiveness of anti-PD1/PD-L1 therapies (26).

Monoclonal antibodies labeled with PET or SPECT isotopes have been able to image tumor molecular targets preclinically, including PD-L1 and other immunooncology targets (27-30). Monoclonal antibody PET imaging often leads to tumor-to-blood ratios in excess of 3:1 in some mouse xenografts models but requires imaging to be performed several days after tracer injection (31). Smaller PD-L1binding ligands with clearance, distribution, and targeting temporal characteristics more closely matched to the half-life of ${ }^{18} \mathrm{~F}$ are clinically desirable because they enable imaging a few hours after tracer injection, making it logistically easier to incorporate imaging in therapeutic trials. Affibody ligands, with their relatively fast clearance and short targeting times, have shown promise as ${ }^{18} \mathrm{~F}$-based PET imaging agents in preclinical studies $(13,32)$.

${ }^{18} \mathrm{~F}-\mathrm{AlF}-\mathrm{NOTA}$ labeling provides a straightforward and reliable 1-step labeling method for ligands that can withstand high temperatures without losing their folding structure (13,33). An initial study of the ${ }^{18} \mathrm{~F}$-AlF -NOTA-PRGD2 peptide binding $\alpha-v-\beta-3$ has shown favorable clinical biodistribution and tumor targeting (34). In this study, satisfactory radiochemical purity, yield, and specific activity in excess of 14.6 GBq/ $\mu \mathrm{mol}$ allowed in vivo static imaging and ex vivo biodistribution with injected activities of about $400 \mathrm{kBq}$ per subject. Doses of the injected tracer were kept at approximately 27 pmol to avoid self-block, which was a concern given the relatively low LOX tumor PD-L1 concentration of approximately $4.9 \mathrm{nM}$. Because the typical peak tumor \% ID/g observed was about 3, Affibody ligand concentrations in tumors were kept under approximately $0.4 \mathrm{nM}$, well below target saturation levels.

It is likely that the low tracer uptake in most tissues in mice is partly driven by lack of cross-reactivity of ${ }^{18} \mathrm{~F}$-AlF-NOTA$\mathrm{Z}_{\mathrm{PD}-\mathrm{L} 1 \_1}$ with mouse PD-L1. In clinical imaging, binding to tissues that normally express PD-L1 may lead to a different 
background distribution and clearance than in the mouse. $\mathrm{Z}_{\mathrm{PD}-\mathrm{L1} 1 \text { 1 }}$ cross-reacts with rhesus monkey PD-L1 with an affinity similar to human PD-L1 (Fig. 1), allowing for the possibility of labeling optimization through imaging studies in nonhuman primates, where PD-L1 background signal should resemble that of human subjects. Kidney retention is likely due to tubular reuptake of proteins, where ${ }^{18} \mathrm{~F}$-AlF label is trapped after cleavage of the Affibody ligand. High clinical kidney radioisotope retention would limit the injected activity due to dosimetry considerations, resulting in increased image noise with lower sensitivity to target-positive lesions. The use of charged linkers coupled with nonresidualizing fluorinated labels such as 18FBA has shown low kidney and liver retention in a xenograft mouse model (35). PET images of spine, joints, and skull show some uptake indicative of defluorination, which could be mitigated by the use of other labeling approaches (13).

The uptake of ${ }^{18} \mathrm{~F}-\mathrm{AlF}-\mathrm{NOTA}-\mathrm{Z}_{\mathrm{PD}-\mathrm{L} 1 \_1}$ in the LOX xenograft was lower than reported for other tracers targeting similarly lowexpressing proteins in tumors (36), including a peptidic anti-PDL1 tracer (37). It is possible that the unusually fast blood clearance of ${ }^{18} \mathrm{~F}-\mathrm{AlF}-\mathrm{NOTA}-\mathrm{Z}_{\mathrm{PD}-\mathrm{L} 1 \_1}$ led to low target tissue uptake but high tumor-to-blood ratios. Future studies will explore the kinetics of the Affibody tracer and its relationship to tumor uptake.

\section{CONCLUSION}

The Affibody ligand ${ }^{18} \mathrm{~F}-\mathrm{AlF}-\mathrm{NOTA}-\mathrm{Z}_{\mathrm{PD}-\mathrm{L} 1 \_1}$ showed fast blood clearance and specific targeting in vivo, with low background in all normal tissues except the kidneys. Modifications to the linker or method of radiolabel incorporation along with increases in ligand affinity should lead to diminished renal and bone uptake and greater tumor signal, resulting in a higher probability of clinical wholebody PD-L1 positive tumor detection.

\section{REFERENCES}

1. Taube JM, Klein AP, Brahmer JR, et al. Association of PD-1, PD-1 ligands, and other features of the tumor immune microenvironment with response to anti-PD1 therapy. Clin Cancer Res. 2014;20:5064-5074.

2. Garon EB, Rizvi NA, Hui R, et al. Pembrolizumab for the treatment of nonsmall-cell lung cancer. N Engl J Med. 2016;372:2018-2028.

3. Herbst RS, Baas P, Kim D-W, et al. Pembrolizumab versus docetaxel for previously treated, PD-L1-positive, advanced non-small-cell lung cancer (KEYNOTE-010): a randomised controlled trial. Lancet. 2015;387:1540-1550.

4. Lipson EJ. Re-orienting the immune system: durable tumor regression and successful re-induction therapy using anti-PD1 antibodies. OncoImmunology. 2013;2:e23661.

5. Frederick DT, Piris A, Cogdill AP, et al. BRAF inhibition is associated with enhanced melanoma antigen expression and a more favorable tumor microenvironment in patients with metastatic melanoma. Clin Cancer Res. 2013;19:1225-1231.

6. Dijkers EC, Munnik THO, Kosterink JG, et al. Biodistribution of ${ }^{89} \mathrm{Zr}$-trastuzumab and PET imaging of HER2-positive lesions in patients with metastatic breast cancer. Clin Pharmacol Ther. 2010;87:586-592.

7. Börjesson PKE, Jauw YWS, Boellard R, et al. Performance of immuno-positron emission tomography with zirconium-89-labeled chimeric monoclonal antibody U36 in the detection of lymph node metastases in head and neck cancer patients. Clin Cancer Res. 2006:12:2133-2140.

8. Zhang Y, Hong H, Cai W. Pet tracers based on zirconium-89. Curr Radiopharm. 2011;4:131-139.

9. Sundaresan G, Yasaki PJ, Shively JE, et al. ${ }^{124}$ I-Labeled engineered anti-CEA minibodies and diabodies allow high-contrast, antigen-specific small-animal PET imaging of xenografts in athymic mice. J Nucl Med. 2003;44:1962-1969.

10. Divgi CR, O'Donoghue JA, Welt S, et al. Phase I clinical trial with fractionated radioimmunotherapy using ${ }^{131} \mathrm{I}$-labeled chimeric G250 in metastatic renal cancer. J Nucl Med. 2004;45:1412-1421.

11. Jauw YW, Menke-van der Houven van Oordt CW, Hoekstra OS, et al. Immunopositron emission tomography with zirconium-89-labeled monoclonal antibodies in oncology: what can we learn from initial clinical trials? Front Pharmacol. 2016;7:131.

12. Löfblom J, Feldwisch J, Tolmachev V, Carlsson J, Stahl S, Frejd FY. Affibody molecules: engineered proteins for therapeutic, diagnostic and biotechnological applications. FEBS Lett. 2010;584:2670-2680.

13. Glaser M, Iveson P, Hoppmann S, et al. Three methods for ${ }^{18} \mathrm{~F}$ labeling of the HER2-binding affibody molecule ZHER2:2891 including preclinical assessment. J Nucl Med. 2013;54:1981-1988.

14. Kramer-Marek G, Shenoy N, Seidel J, Griffiths GL, Choyke P, Capala J. ${ }^{68} \mathrm{Ga}-$ DOTA-affibody molecule for in vivo assessment of HER2/neu expression with PET. Eur J Nucl Med Mol Imaging. 2011;38:1967-1976.

15. Heskamp S, Laverman P, Rosik D, et al. Imaging of human epidermal growth factor receptor type 2 expression with ${ }^{18} \mathrm{~F}$-labeled affibody molecule ZHER2:2395 in a mouse model for ovarian cancer. $J$ Nucl Med. 2012;53:146-153.

16. Strand J, Honarvar H, Perols A, et al. Influence of macrocyclic chelators on the targeting properties of ${ }^{68} \mathrm{Ga}$-labeled synthetic affibody molecules. PLoS One. 2013;42:446-454

17. Sörensen J, Sandberg M, Wennborg A, et al. First-in-Human molecular imaging of HER2 expression in breast cancer metastases using the ${ }^{111}$ In-ABY-025 affibody molecule. J Nucl Med. 2014;55:730-735.

18. Baum RP, Prasad V, Muller D, et al. Molecular imaging of HER2-expressing malignant tumors in breast cancer patients using synthetic ${ }^{111} \mathrm{In}$ - or ${ }^{68} \mathrm{Ga}$-labeled affibody molecules. J Nucl Med. 2010;51:892-897.

19. Sörensen J, Velikyan I, Sandberg D, et al. Measuring HER2-receptor expression in metastatic breast cancer using [68Ga]ABY-025 affibody PET/CT. Theranostics. 2016;6:262-271.

20. Dong Y, Richards JA, Gupta R, et al. PTEN functions as a melanoma tumor suppressor by promoting host immune response. Oncogene. 2014;33:4632-4642.

21. Andorsky DJ, Yamada RE, Said J, Pinkus GS, Betting DJ, Timmerman JM. Programmed death ligand 1 is expressed by non-hodgkin lymphomas and inhibits the activity of tumor-associated T cells. Clin Cancer Res. 2011;17:4232-4244.

22. Le DT, Uram JN, Wang H, et al. PD-1 blockade in tumors with mismatch-repair deficiency. N Engl J Med. 2015;372:2509-2520.

23. Zou W, Chen L. Inhibitory B7-family molecules in the tumour microenvironment. Nat Rev Immunol. 2008;8:467-477.

24. Topalian SL, Hodi SF, Brahmer JR, et al. Safety, activity, and immune correlates of anti-PD-1 antibody in cancer. N Engl J Med. 2012;366:2443-2454.

25. Powles T, Eder JP, Fine GD, et al. MPDL3280A (anti-PD-L1) treatment leads to clinical activity in metastatic bladder cancer. Nature. 2014;515:558-562.

26. O'Donnell JS, Long GV, Scolyer RA, Teng MW, Smyth MJ. Resistance to PD1/ PDL1 checkpoint inhibition. Cancer Treat Rev. 2017;52:71-81.

27. Heskamp S, Hobo W, Mokernboer-Kuenen JDM, et al. Noninvasive imaging of tumor PD-L1 expression using radiolabeled anti-PD-L1 antibodies. Cancer Res. 2015;75:2928-2936.

28. Josefsson A, Nedrow JR, Park S, et al. Imaging, biodistribution, and dosimetry of radionuclide-labeled PD-L1 antibody in an immunocompetent mouse model of breast cancer. Cancer Res. 2016;76:472-479.

29. Ehlerding EB, England CG, McNeel DG, Cai W. Molecular imaging of immunotherapy targets in cancer. J Nucl Med. 2016;57:1487-1492.

30. Hettich M, Braun F, Bartholoma MD, Schirmbeck R, Niedermann G. Highresolution PET Imaging with therapeutic antibody-based PD-1/PD-L1 checkpoint tracers. Theranostics. 2016;6:1629-1640.

31. van de Watering FC, Rijpkema M, Perk L, Brinkmann U, Oyen WJ, Boerman OC. Zirconium-89 labeled antibodies: a new tool for molecular imaging in cancer patients. Biomed Res Int. 2014;2014:203601.

32. Kramer-Marek G, Kiesewetter DO, Martiniova L, Jagoda E, Lee SB, Capala J. $\left[{ }^{18}\right.$ F]FBEM-ZHER2:342-affibody molecule: a new molecular tracer for in vivo monitoring of HER2 expression by positron emission tomography. Eur J Nucl Med Mol Imaging. 2008;35:1008-1018.

33. McBride WJ, D'Souza CA, Sharkey RM, et al. Improved ${ }^{18} \mathrm{~F}$ labeling of peptides with a fluoride-aluminum-chelate complex. Bioconjug Chem. 2010;21:1331-1340.

34. Wan W, Guo N, Pan D, et al. First experience of ${ }^{18} \mathrm{~F}$-alfatide in lung cancer patients using a new lyophilized kit for rapid radiofluorination. J Nucl Med. 2013;54:691-698.

35. Rosik D, Thibblin A, Antoni G, et al. Incorporation of a triglutamyl spacer improves the biodistribution of synthetic affibody molecules radiofluorinated at the N-terminus via oxime formation with ${ }^{18} \mathrm{~F}-4$-fluorobenzaldehyde. Bioconjug Chem. 2014;25:82-92.

36. Tolmachev V, Wallberg H, Sandstrom M, Hansson M, Wennborg A, Orlova A. Optimal specific radioactivity of anti-HER2 Affibody molecules enables discrimination between xenografts with high and low HER2 expression levels. Eur J Nucl Med Mol Imaging. 2011;38:531-539.

37. Chatterjee S, Lesniak WG, Miller MS, et al. Rapid PD-L1 detection in tumors with PET using a highly specific peptide. Biochem Biophys Res Commun. 2017;483:258-263. 